\title{
Pseudomesotheliomatous Carcinoma due to Pleural Metastasis from Renal Pelvic Cancer
}

\author{
Yuki Katsuya ${ }^{1}$, Munehisa Fukusumi ${ }^{1}$, Shigeki Morita ${ }^{2}$, Tatsuya Ibe ${ }^{1}$, Kazushige Wakuda ${ }^{1}$, \\ Atsuto Mouri ${ }^{1}$, Yoichiro Hamamoto ${ }^{1}$, Kazuaki Yamada ${ }^{3}$ and Mitsuhiro Kamimura ${ }^{1}$
}

\begin{abstract}
A 78-year-old man was referred to our department with a one-week history of dyspnea and coughing. A chest X-ray showed massive left pleural effusion. Computed tomography revealed diffuse irregular thickening of the left pleura similar to malignant mesothelioma and multiple nodules in both lungs. The patient died of respiratory failure nine days after hospitalization. An autopsy revealed metastasis to the pleura and lungs from urothelial carcinoma of the left kidney.
\end{abstract}

Key words: pseudomesotheliomatous carcinoma, renal pelvic cancer

(Intern Med 53: 871-874, 2014)

(DOI: 10.2169/internalmedicine.53.1309)

\section{Introduction}

Pseudomesotheliomatous carcinoma is a malignant tumor that extends along the pleura similar to malignant mesothelioma. Almost all cases originate from lung cancer, particularly adenocarcinoma. However, pseudomesotheliomatous progression rarely occurs in pleural metastasis from breast, ovarian, gastrointestinal, renal or urinary tract cancer $(1,2)$. We herein report the case of a Japanese man who died of pseudomesotheliomatous carcinoma from renal pelvic cancer.

\section{Case Report}

A 78-year-old man was admitted to our department for an evaluation and treatment of massive left pleural effusion on a chest X-ray. He had a one-week history of dyspnea, coughing and loss of appetite. He had no previous asbestos exposure or smoking history. Regarding the patient's blood count and blood chemistry, the white blood cell count was $15,300 / \mu \mathrm{L}$, the C-reactive protein level was $20.4 \mathrm{mg} / \mathrm{dL}$, the creatinine level was $1.75 \mathrm{mg} / \mathrm{dL}$, the squamousceHcarcbomarelatedantigen (SCC) level was $250 \mathrm{ng} / \mathrm{mL}$ and the cytokeratin 19 fragment (CYFRA) level was $1.7 \mathrm{ng} / \mathrm{mL}$. The pleural effusion was exudative, and the level of hyaluronic acid was 40,100 ng/mL. Cytology of the fluid revealed a class III status. Computed tomography (CT) demonstrated diffuse thickening of the left pleura, multiple areas of nodular density in both lung fields and an irregular mass measuring $6.0 \times 5.0 \mathrm{~cm}$ in the left kidney (Fig. 1). A urinalysis showed no proteinuria or hematuria. A thoracoscopic pleural biopsy was scheduled; however, the patient's condition rapidly deteriorated. On the ninth day after admission, he died of respiratory and cardiovascular failure and disseminated intravascular coagulation.

On autopsy, a $6.5-\mathrm{cm}$ sized, whitish, irregular solid tumor was detected on the upper middle part of the left kidney (Fig. 2). The left pleura was circumferentially thickened along with the tumor, a part of which had infiltrated the lung parenchyma. Histopathology showed poorly differentiated transitional cell carcinoma and comorbid squamous cell carcinoma in both the renal pelvis and lung lesions (Fig. 3). Therefore, a diagnosis of urothelial carcinoma of the left kidney (grade 3, stage IV) with metastasis to the left pleura and lung was established.

\footnotetext{
${ }^{1}$ Department of Pulmonary Medicine, National Hospital Organization Disaster Medical Center, Japan, ${ }^{2}$ Department of Pathology, Graduate School of Medicine, The University of Tokyo, Japan and ${ }^{3}$ Department of Pathology, National Hospital Organization Disaster Medical Center, Japan

Received for publication July 5, 2013; Accepted for publication November 4, 2013

Correspondence to Dr. Munehisa Fukusumi, munesumi@hotmail.com
} 

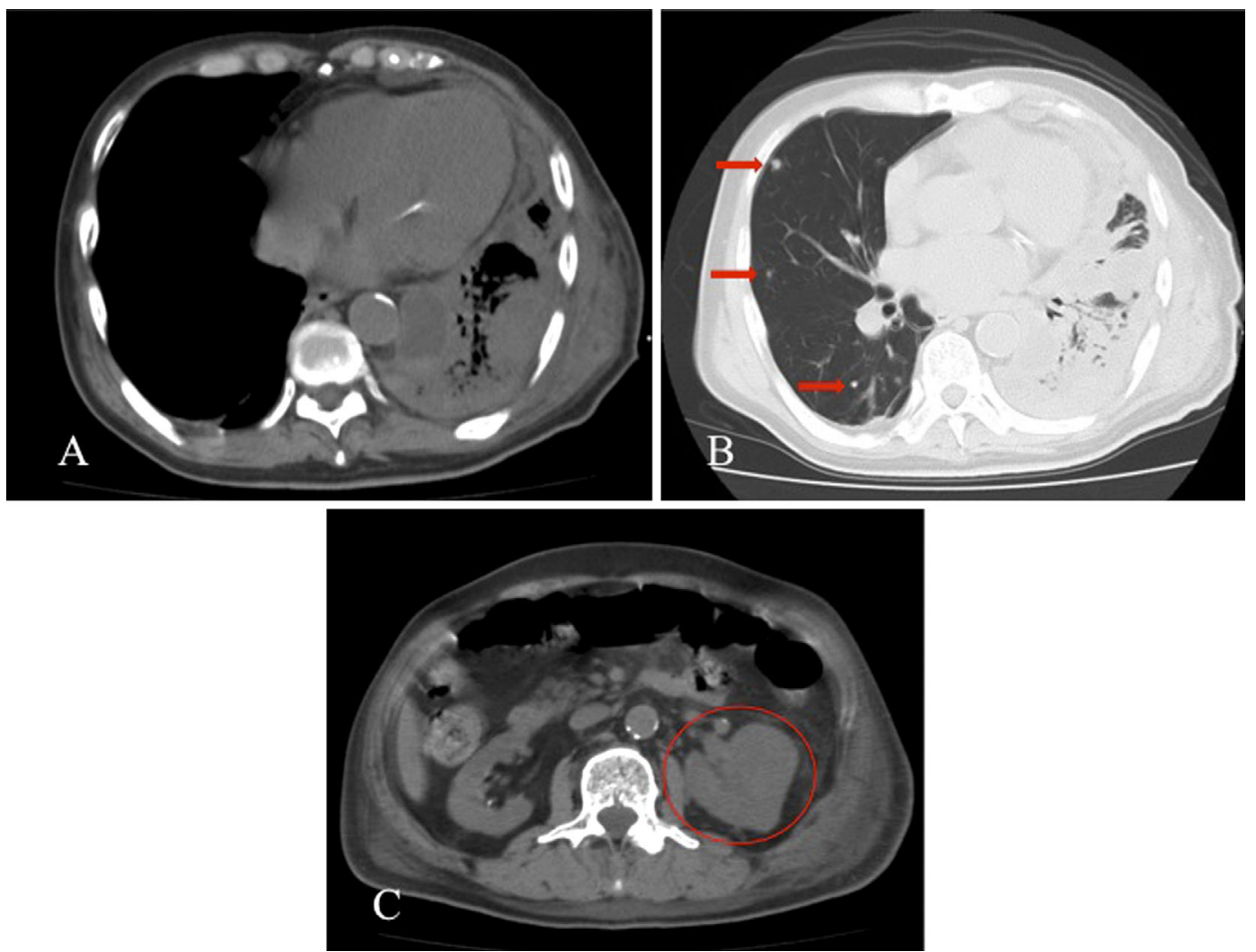

Figure 1. A, B: CT revealed irregular extensive left pleural thickening and small nodules (arrows) in the right lung. $\mathrm{C}$ : The left kidney was irregularly swollen (circle).

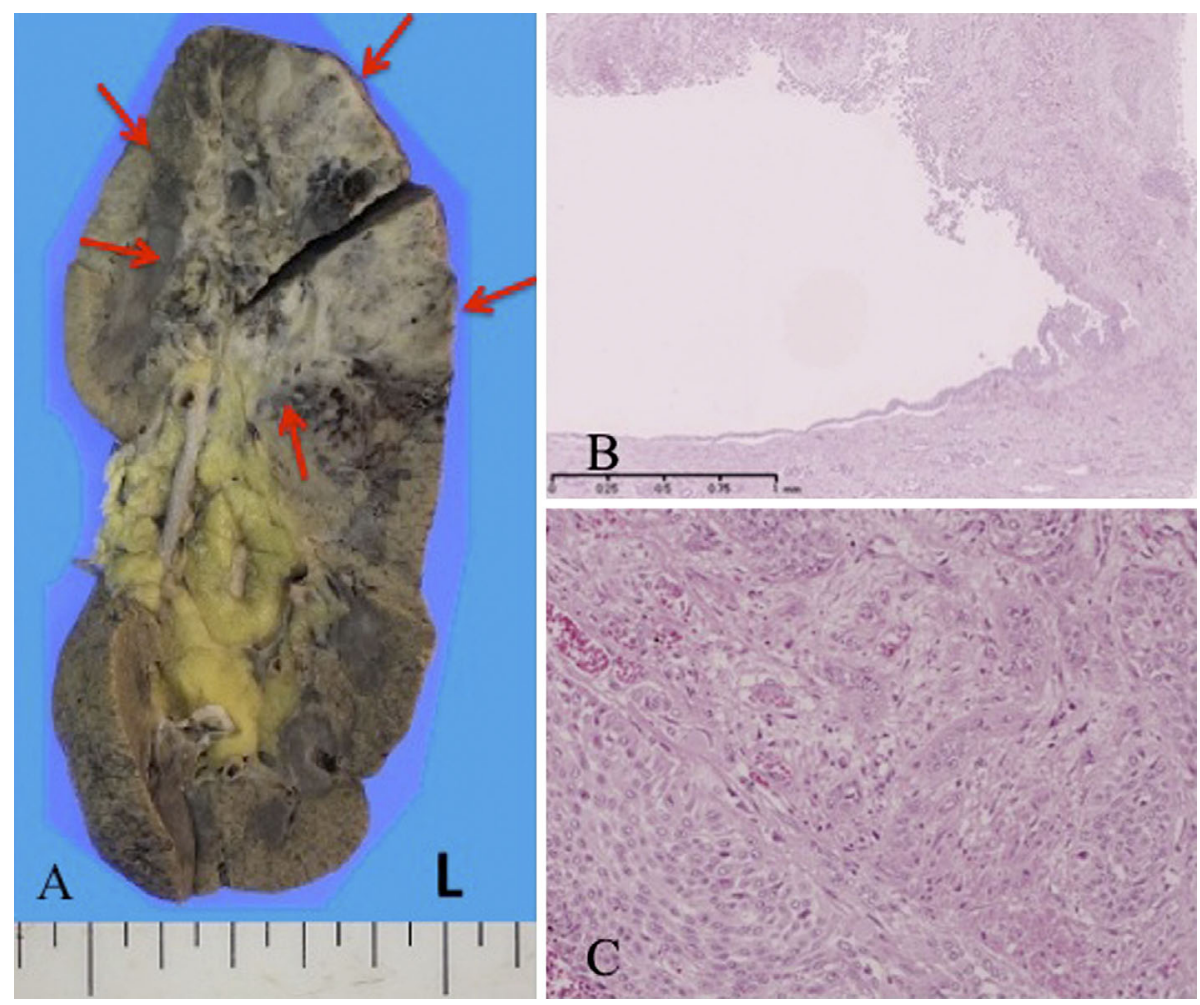

Figure 2. A: The gross appearance showed a solid tumor in the upper middle area of the left kidney (arrows). B, C: A photomicrographic image of the primary lesion (Hematoxylin and Eosin staining; original magnification $\times 25, \times 100$ ) showing poorly differentiated transitional cell carcinoma displacing normal urothelial cells. 

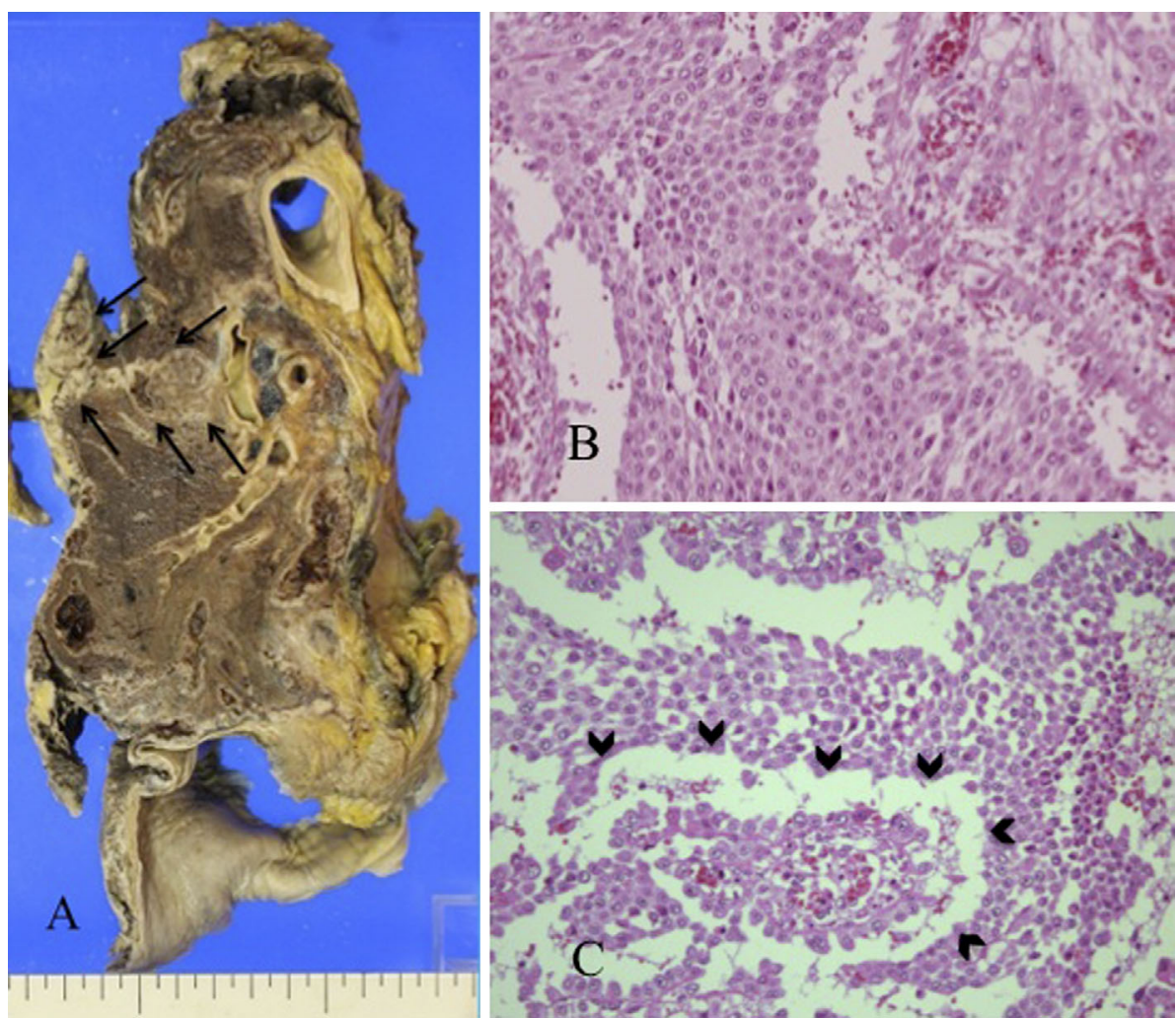

Figure 3. A: The gross appearance showed diffuse pleural involvement by the tumor, which had invaded the left lung parenchyma, and intrapulmonary hematogenous metastasis (arrows). B, C: A photomicrographic image of the lung lesion directly invading the pleura $(B)$ with intrapulmonary hematogenous metastasis (C) (Hematoxylin and Eosin staining; original magnification $\times 200$ ) showing that the histological features of the tumor cells in the lung were similar to those of the primary lesion in the renal pelvis. The tumor cells had invaded through the bloodstream (arrowheads).

\section{Discussion}

A diffuse growth pattern of a pleural neoplasm radiologically mimicking malignant mesothelioma, so-called 'pseudomesotheliomatous carcinoma, was first reported by Harwood et al. (3) Attanoos reported the most common primary site for pseudomesotheliomatous pleural metastasis to be peripheral lung cancer $(88 \%)$ ). Other primary sites include transitional cell carcinoma of the bladder (4\%), renal cell carcinoma (2\%), ductal pancreatic adenocarcinoma (2\%), prostatic adenocarcinoma (2\%) and so on (1); such tumors are associated with a poor prognosis (1). In patients with pseudomesotheliomatous carcinomas, diffuse thickening of the pleura, pleural effusion and nodules in the lungs are common features, although pleural plaque is less common than in patients with malignant mesothelioma (1). Differentiating between pseudomesotheliomatous carcinoma and malignant mesothelioma based on appearance only is sometimes difficult. Therefore, the combination of imaging and pathological findings is essential for diagnosis. Immunohistochemistry is an effective method, and the level of carcinoembryonic antigen has been found to be the most specific marker for adenocarcinoma (1). The thyroid transcription factor-1 level has also been shown to be a useful marker for primary lung adenocarcinoma (4). On the other hand, the calretinin level has been demonstrated to be a highly sensitive and specific marker for mesothelioma (1).

Transitional cell carcinoma of the renal pelvis accounts for only $5-6 \%$ of all urothelial tumors. Distant metastasis to the lungs, liver and colon is frequently recognized (5), although metastasis to the cardiac chamber $(6,7)$, cerebrum (8) and skin (9) rarely occurs. However, there are no reported cases of pseudomesotheliomatous carcinoma originating from transitional cell carcinoma of the renal pelvis. In the present case, we suppose that the cancer metastasized hematogenously, as the autopsy revealed invasion of the cancer to the renal vein without lymph node metastasis in either the abdominal or thoracic cavity.

In conclusion, in the present case, transitional cell carcinoma of the renal pelvis diffusely metastasized to the pleura, which manifested with mesothelioma-like findings. Although rare, renal pelvic cancer should be taken into account as a primary site of pseudomesotheliomatous carcinoma.

The authors state that they have no Conflict of Interest (COI). 


\section{References}

1. Attanoos RL, Gibbs AR. 'Pseudomesotheliomatous' carcinomas of the pleura: a 10-year analysis of cases from the Environmental Lung Disease Research Group, Cardiff. Histopathology 43: 444452, 2003.

2. Leers MP, Aarts MM, Theunissen PH. E-cadherin and calretinin: a useful combination of immunochemical markers for differentiation between mesothelioma and metastatic adenocarcinoma. Histopathology 32: 209-216, 1998.

3. Harwood TR, Gracey DR, Yokoo H. Pseudomesotheliomatous carcinoma of the lung. A variant of peripheral lung cancer. Am J Clin Pathol 65: 159-167, 1976.

4. Reis-Filho JS, Carrilho C, Valenti C, et al. Is TTF1 a good immunohistochemical marker to distinguish primary from metastatic lung adenocarcinomas? Pathol Res Pract 196: 835-840, 2000.
5. Tan LB, Chang LL, Cheng KI, Huang CH, Kwan AL. Transitional cell carcinomas of the renal pelvis and the ureter: comparative demographic characteristics, pathological grade and stage and 5year survival in a Taiwanese population. BJU Int 103: 312-316, 2009.

6. Sobczyk D, Nosal M, Myc J, et al. Cardiac metastasis due to pulmonary metastasis from a transitional cell carcinoma. Eur J Echocardiogr 9: 113-115, 2008.

7. Murakami T, Komiya A, Mikata K, Kaneko S, Ikeda I. Cardiac metastasis of renal pelvic cancer. Int J Urol 14: 240-241, 2007.

8. Turgut M, Akyüz O, Kaçar F. Solitary cerebral metastasis from transitional cell carcinoma of the urinary tract. J Clin Neurosci 14: 1129-1132, 2007.

9. Lin CY, Lee CT, Huang JS, Chang LC. Transitional cell carcinoma metastasis to arm skin from the renal pelvis. Chang Gung Med J 26: 525-529, 2003.

(C) 2014 The Japanese Society of Internal Medicine http://www.naika.or.jp/imonline/index.html 